\title{
Determination of elastic constants of Inconel-625 superalloy, using laser-based ultrasonic
}

\author{
Anita Shukla ${ }^{1}$ (1)
}

Received: 18 July 2018 / Accepted: 26 November 2018 / Published online: 29 November 2018

(c) The Author(s) 2018

\begin{abstract}
This paper deals with the determination of the elastic constants of Inconel-625 from the analysis of laser-generated ultrasonic bulk waves. A pulsed Nd/YAG laser $(1064 \mathrm{~nm})$ is used for ultrasonic generation in a thick stepped Inconel-625 sample, and a He-Ne laser is used for heterodyne detection of the laser-generated signals. Ultrasonic signals obtained at epicenter and at off-epicenter position of the detection points are analyzed using wavelet transforms. Here, the identification of pressure as well as shear waves and the estimation of their velocities are done successfully. From the estimated velocities, the elastic constants (Young's modulus, shear modulus, bulk modulus, Lames constant and Poisson's ratio) are calculated. The agreement of these constants with the standard values confirms the identification of shear waves at off-epicenter position. The work presented in this paper brings out the applicability of the study of laser-generated bulk waves for the determination of elastic constants of any bulk material.
\end{abstract}

Keywords Laser-based ultrasonics (LBU) · Wavelet transforms · Pressure waves · Shear waves · Elastic constants · Inconel

\section{Introduction}

Inconel alloys possess several properties making them well suited for engineering applications in extreme environments. Inconel is very resistant to oxidation and corrosion, when heated it forms a thick passivating oxide layer, protecting it from further attack. Inconel retains its strength over a wide range of temperatures. This makes it particularly attractive in high-temperature applications where aluminum and steel would soften. It is a high-performance alloy. Inconel has wide application in the areas of aerospace industry, jet engines and turbine blades, etc. Determination of internal structure and material properties of an object without actually destructing or damaging the material is achieved using nondestructive evaluation (NDE) techniques.

The several NDE techniques available are being discussed here in brief. Radiographic inspection (X-ray and gammaray) involves penetration of gamma- or X-ray radiation on materials and products to detect defects or examine internal or hidden features. In acoustic emission testing, a localized

Anita Shukla

shukla.anita27@gmail.com

1 Pranveer Singh Institute of Technology, Kanpur, India external force is applied to the part being tested. The resulting stress waves in turn generate short-lived, high-frequency elastic waves that are detected by sensors that have been attached to the part surface. Ultrasonic inspection uses the same principle as is used in naval SONAR and fish finders. Ultra-high-frequency sound is introduced into the part being inspected and by measuring the returning time of reflected wave, the distance to the reflector (the indication with the different acoustic impedance) can be determined. Neutron radiography uses an intense beam of low-energy neutrons as a penetrating medium. Neutrons generated by linear accelerators, betatrons or other sources penetrate most metallic materials, rendering them transparent, but are attenuated by most organic materials (including water, due to its high hydrogen content) which allows those materials to be seen within the component being inspected. Magnetic particle testing (MT) uses one or more magnetic fields to locate surface and near-surface discontinuities in ferromagnetic materials. This produces a visible indication of defect on the surface of the material.

Among the several NDE techniques available, laser-based ultrasonic (LBU) is gaining importance in the recent times. In LBU, a high-intensity laser beam is used to generate ultrasound in the test object. One of the biggest advantages of LBU technique is that generation and detection of the 
ultrasound can be made at a distance, without any physical contact with the surface of the component to be inspected and furthermore no coupling medium is required. However, laser generation of ultrasound has the disadvantage of simultaneous generation of different waves such as shear and longitudinal bulk waves as well as Rayleigh and Lamb waves, thus complicating the process of signal analysis.

Scruby and Drain [1] discussed the mechanisms of generating various ultrasonic waves with lasers and detection by using variety of laser interferometers. Laser irradiation of bulk samples leads to the generation of pressure (longitudinal) and shear (transverse) waves, while it leads to the generation of Lamb waves in thin plates. Scruby et al. [2] carried out the quantitative studies on thermally generated elastic waves in laser-irradiated metals. Wavelet analysis and the properties of different wavelets are explained by Daubechies [3] in her work. Georgiou [4] discussed tissue characterization using the continuous wavelet transform in his work. Singhal et al. [5] discussed recent studies in aluminum using LBU and presented bulk wave signal analysis using wavelet transforms, while Pramila et al. [6] presented the frequency analysis of laser-generated pressure waves in aluminum. Pramila et al. [7] in their recent work studied pressure wave behavior using LBU in Inconel superalloy using wavelet transforms. Zhang et al. [8] measured the shear wave velocities in metals using specially designed speckle interferometer. Ultrasonic velocity studies and laser processing of Inconel are reported by Kumar et al. [9] and Bugayev et al. [10], respectively. Apart from LBU, there are other NDE methods available for characterization of Inconel 625 which have their own merits and demerits. Rai et al. [11] studied the microstructures in Inconel 625 by employing X-ray diffraction peak broadening method, another NDE method for the characterization of microstructures in Inconel 625.

The present work deals with laser-generated bulk waves both shear and pressure, in Inconel-625 and their analysis using wavelet transforms. Inconel-625 is also prominently known for its unique combination of yield strength, tensile strength, fatigue strength, creep strength and excellent weldability. Inconel 625 is a nickel-chromium-molybdenum alloy with an addition of niobium that acts with the molybdenum to stiffen the alloy's matrix and thereby provide high strength without a strengthening heat treatment. The alloy resists a wide range of severely corrosive environments and is especially resistant to pitting and crevice corrosion. It has been widely used in chemical processing, aerospace and marine engineering, pollution control equipment and nuclear reactors.

Fabrication of nickel-based superalloy components could be done in two ways first, they can be made as an integrated casting, and second, the parts can be joined to form the final assembly. Components made from nickel-based superalloy when exposed to harsh operating environments such as high temperatures, oxidizing and reducing conditions for longer periods give rise to cracks that propagate through the surface irregularities and cause failure of the parts. Thus, it becomes important to measure elastic constants of Inconel 625.

In the present work, the elastic constants of Inconel 625 have been calculated using wavelet transforms. The wavelet transforms are generated using MATLAB software. Results thus obtained are used for the estimation of elastic constants.

\section{Experimental details}

The schematic layout of experimental setup is shown in Fig. 1. A pulsed Nd/YAG laser is used to generate ultrasonic waves in a stepped sample of Inconel-625. The $1064 \mathrm{~nm}$ laser line with $7 \mathrm{~ns}$ pulse width was used for signal generation. The laser energy used was $200 \mathrm{~mJ}$. A heterodyne laser interferometer $(\mathrm{He}-\mathrm{Ne}, 633 \mathrm{~nm})$ is used for signal detection [1].

The signals are amplified and digitized using a digital oscilloscope. Recorded waveforms on the oscilloscope are transferred to a computer over an USB/ethernet interface for subsequent storage and analysis.

In the present work, only one stepped sample was prepared with the help of laboratory technicians at IITK. The width of the sample was kept $34.5 \mathrm{~mm}$, while the height was $45 \mathrm{~mm}$. The step size was taken $10 \mathrm{~mm}$. The schematic representation of stepped sample is shown in Fig. 2. The geometry of the sample is such that the center point of each step, where the source laser impinges, lies on the arc of a circle. This is to ensure that time of flight of the bulk waves transmitted to the other side is constant for all angles of incidence. This geometry of the sample simplifies the velocity calculation as times of flight for different waves remain constant, and it also eliminates the need to take the attenuation effects into consideration while making calculations using signal intensities.

In the experimental arrangement using laser heterodyne detection, it is necessary that the probe laser beam should get back reflected. The heterodyne laser detects disturbances along the laser beam direction. If the ultrasonic waves generated in material are detected at "epicenter" as shown in Fig. 3a, then one can only detect the pressure wave displacements.

However, if the ultrasonic signal is picked up at off-epicenter position as shown in Fig. 3b, with the probe beam normal to the surface, the heterodyne will be able to pick up the components of vibrational amplitudes of both pressure wave and shear (horizontal) wave.

Laser-generated ultrasonic signals for Inconel stepped sample are recorded repeatedly in the epicenter as well as five off-epicentral directions $\left(10^{\circ}, 20^{\circ}, 30^{\circ}, 40^{\circ}, 50^{\circ}\right)$. 
Fig. 1 Experimental setup for Inconel-625 using LBU

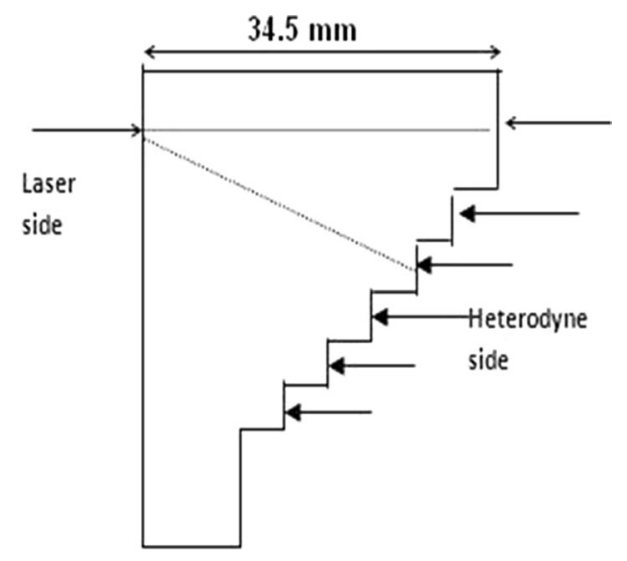

Fig. 2 Stepped sample of Inconel-625

\section{Results and discussion}

The recorded ultrasonic signals are analyzed using wavelet transforms [3]. The details of the analysis are given in the following subsections.

\section{Wavelet transforms}

Jean Morlet first proposed the concept of wavelets in its present theoretical form. A wavelet is a waveform of effectively limited duration that has an average value of zero. Wavelet transform is a useful tool for the interpretation and the analysis of ultrasonic data in the nondestructive
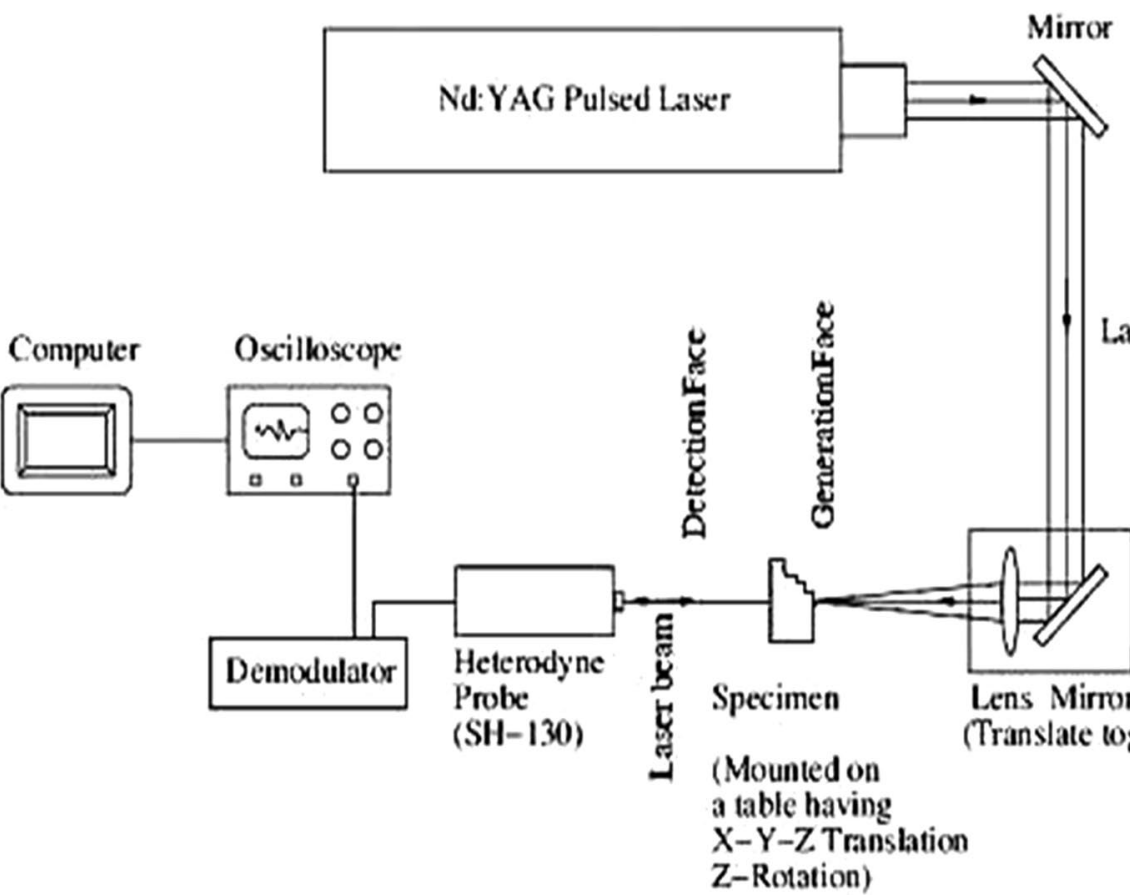

Lasce beam

(a) Ultrasonic wave generation by Nd-YAG laser

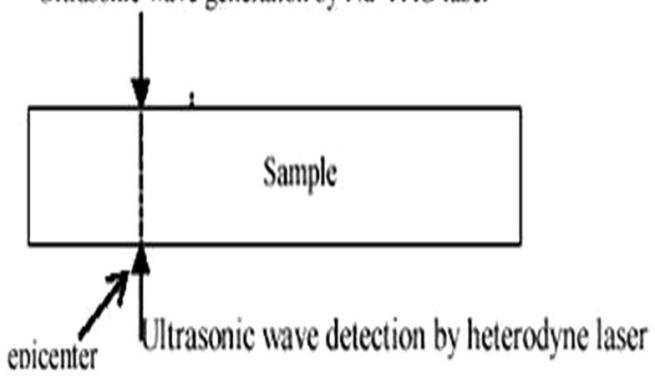

(b) Ultrasonic wave generation by Nd-YAG laser

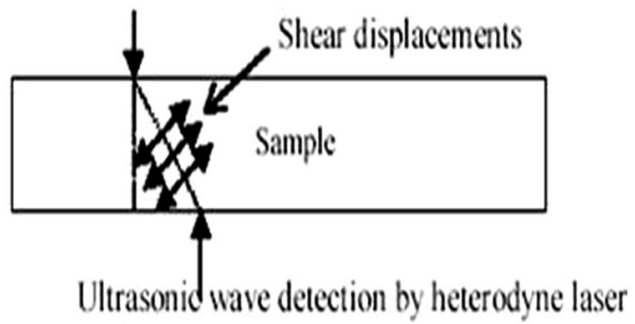

Fig. 3 a Epicenter detection of ultrasonic signals, b off-epicenter detection of ultrasonic signals

evaluation (NDE). Main applications of the wavelet transform are signal analysis in the time-frequency domain, data compression and signal processing. Using wavelet analysis, signals can be characterized in both time and frequency domain simultaneously $[4,5]$. The transform image is plotted as scale versus sample number versus intensity. Wavelet transform of signal obtained for Inconel 


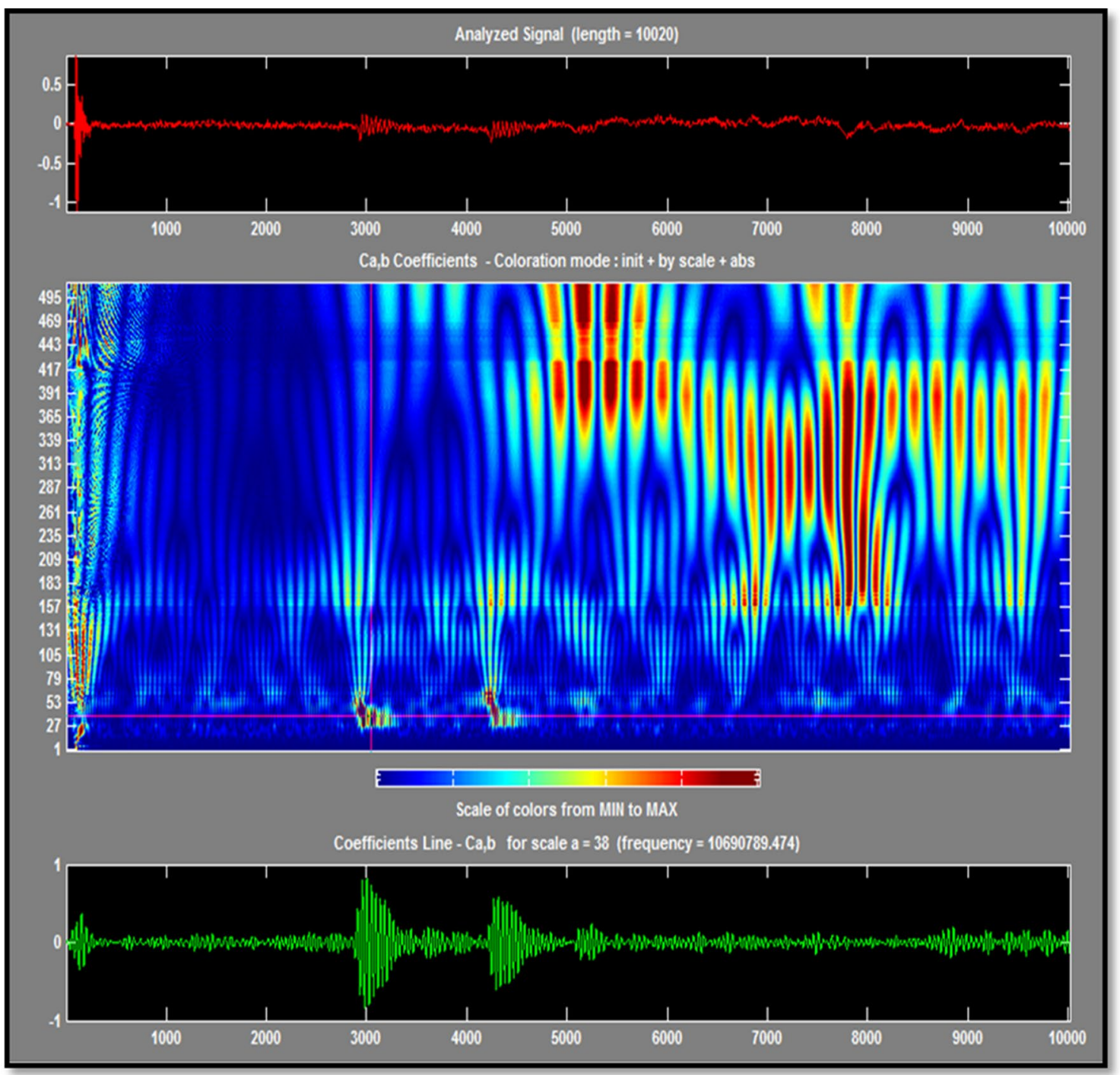

Fig. 4 Wavelet transform of Inconel-625 for detection at epicenter

stepped sample shown in Fig. 4 has three windows. The first window, in the bottom, shows the original signal whose wavelet is to be taken. Second window, in the middle, shows the three-dimensional plot in which $\mathrm{X}$-axis corresponds to sample or time variation, Y-axis referred to as coefficient axis corresponds to frequency according to scale, and Z-axis corresponds to intensity. In this twodimensional plot of the wavelet transform, the variation in intensity is depicted with help of various color schemes. The color scheme in the present figure is such that one can see a gradual change of color from red to blue with various in between colors. The dark blue area corresponds to minimum intensity, while the red area indicates the presence of a strong signal. The third window, on the top, gives temporal distribution of the signal at a particular frequency corresponding to a given scale. By choosing different coefficient lines, one can study the behavior of different frequency components present in a given signal. As the recorded signals contain various frequency components, the wavelet transform of signal can be used to obtain arrival times for different frequency components.

\section{Epicentral detection}

\section{Pressure wave velocity estimation}

The temporal behavior of frequency $10.97 \mathrm{MHz}$ component is shown by the coefficient line in (Fig. 4). It is evident from (Fig. 4) that continuous wavelet transforms give good time-frequency localization as seen by the variation in color representing how dominant a particular frequency component is at a particular time [6]. Among bulk waves, pressure 
wave is known to be having highest velocity so the groups of waves that arrive earliest are identified as the pressure waves. The temporal and spectral behaviors of pressure wave are studied and the results are reported. In this paper, pressure wave velocity and critical angle for Inconel are reported $5823 \mathrm{~m} / \mathrm{s}$ and $30^{\circ}$, respectively. At the critical angle, the pressure wave signal is found to be of minimum intensity.

\section{Off-epicentral detection}

\section{Shear wave velocity estimation}

Figure 5 shows wavelet transform of the LBU signal of Inconel at the critical angle, i.e., $30^{\circ}$. A comparison of Fig. 5 with Fig. 4 reveals that the frequency band in the high-frequency region corresponding to the time of travel of pressure wave is absent in Fig. 5. However, one can see the presence of this high-frequency band at an arrival time of $10.8 \mu$ s. This arrival time can be seen to be almost twice the time of flight of pressure waves as in the case of aluminum [12]. As no other bulk wave signal can be present at this time of arrival, one can assume these to be shear waves. Using the thickness of the sample $(34.5 \mathrm{~mm})$ as distance traveled and the time of arrival, the shear wave velocity is calculated to be $3194 \mathrm{~m} / \mathrm{s}$.

\section{Determination of elastic constants}

Elastic constants (Young's modulus Shear modulus, Bulk's modulus, Lames constant and Poisson's ratio) are functions of ultrasonic velocities. The second column in Table 1 gives the interrelations among these constants. Here, $K=C_{1 /} C_{2}$ and $D$ is the density of Inconel which is taken to be $8.44 \times 10^{3} \mathrm{~kg} /$ $\mathrm{m}^{3}$.

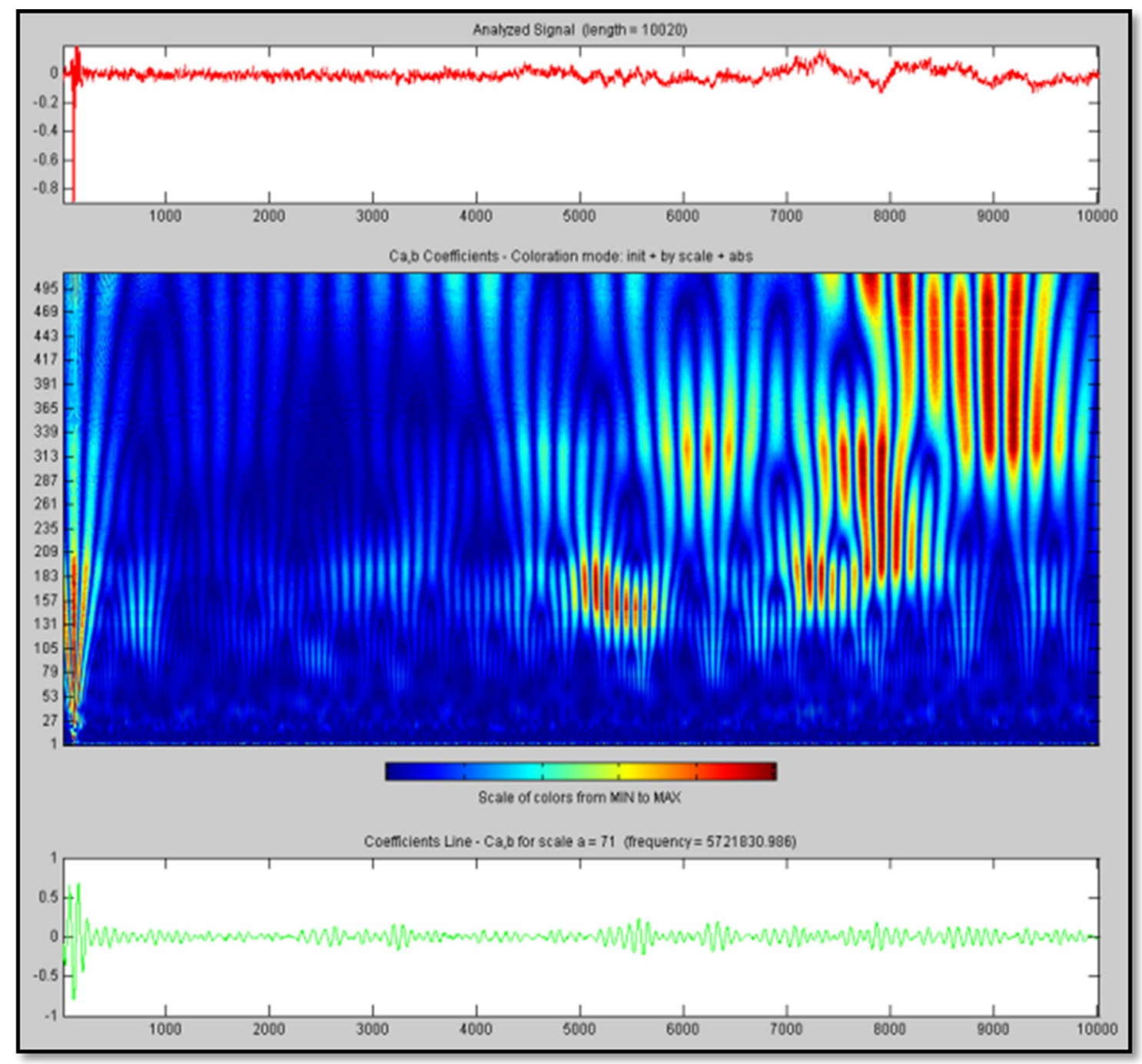

Fig. 5 Wavelet transform of Inconel-625 at the critical angle $\left(30^{\circ}\right)$ 
Table 1 Elastic constants of Inconel-625

\begin{tabular}{llcc}
\hline Name of elastic constant & Formula for elastic constant & $\begin{array}{l}\text { Calculated value }\left(\times 10^{11} \text { dynes/cm } \mathrm{cm}^{2}\right) \\
\text { OR }\left(\times 10^{10} \mathrm{~N} / \mathrm{m}^{2}\right)\end{array}$ & $\begin{array}{l}\text { Standard value } \\
\left(\times 10^{11} \mathrm{dynes} / \mathrm{cm}^{2}\right) \text { OR } \\
\left(\times 10^{10} \mathrm{~N} / \mathrm{m}^{2}\right)\end{array}$ \\
\hline Young's modulus & $Y=D C_{2}^{2}\left(4-3 k^{2}\right) /\left(1-k^{2}\right)$ & 22.09 & 20.8 \\
Shear modulus & $\eta=D C_{2}^{2}$ & 8.6 & 8.1 \\
Bulk modulus & $B=D C_{2}^{2}\left(k^{2}-4 / 3\right)$ & 17.11 & $17.09^{\mathrm{a}}$ \\
Lame's constant & $\lambda=D C_{2}^{2}\left(k^{2}-2\right)$ & 11.37 & $11.82^{\mathrm{a}}$ \\
Poisson's ratio & $\sigma=\left(2-k^{2}\right) /\left(2-2 k^{2}\right)$ & 0.28 & 0.28 \\
\hline
\end{tabular}

${ }^{\text {a }}$ These values are calculated from the reported standard values of $Y$ and $\eta$

Using these relations, the elastic constants are calculated and are given in the third column of Table 1.

The standard values of these constants are given in column 4 [13].

One can see from above table that the values of elastic constants obtained in the present work are in good agreement with the standard values for Inconel. This agreement confirms that the bulk wave signal identified as shear wave in the present work is actually shear wave.

\section{Conclusions}

In the present paper, results of wavelet analysis of LBU signals obtained for stepped sample of Inconel are presented. Identification of pressure as well as shear waves and their velocity estimation is done successfully $[9,10]$.

Accurate match of estimated and standard values of elastic constants confirms the assumed presence of shear waves at off-epicentral position. The technique may be employed for the characterization of different materials in terms of elastic constants.

Open Access This article is distributed under the terms of the Creative Commons Attribution 4.0 International License (http://creativecommons.org/licenses/by/4.0/), which permits unrestricted use, distribution, and reproduction in any medium, provided you give appropriate credit to the original author(s) and the source, provide a link to the Creative Commons license, and indicate if changes were made.

\section{References}

1. Scruby, C.B., Drain, L.E.: Laser ultrasonics: techniques and applications. Adam Hilger Bristol, Philadelphia (1990)
2. Scruby, C.B., Dewhurst, R.J., Hutchins, D.A., Palmer, S.B.: Quantitative studies of thermally generated elastic waves in laser-irradiated metals. J. Appl. Phys. 51, 6210-6216 (1980)

3. Daubechies, I.: Ten lectures on wavelets. In: Society for Industrial and Applied Mathematics, Philadelphia (1992)

4. Georgiou, G., Cohen, F.S.: Tissue characterization using the continuous wavelet transform. I. Decomposition method. IEEE Trans. Ultrason. Ferroelectr. Freq. Control 48, 355-373 (2001)

5. Singhal, M., Pramila, T., Raghuram, V., Kishore, N.N.: Characterization of laser generated bulk waves using wavelet transforms and pattern recognition. In: Asia Pacific Conference on NDT, Auckland, Newzealand (2006)

6. Pramila, T., Shukla, A., Kishore, N.N., Raghuram, V.: In: Proceedings of the HSNT conference, Chania, Greece (2007)

7. Zhang, S.Y., Paul, M., Fassbender, S., Schleichert, U., Arnold, W.: Experimental study of laser-generated shear waves using interferometry. Res. Nondestruct. Eval. 2, 143-155 (1990)

8. Pramila, T., Shukla, A., Raghuram, V.: Studies on laser generated ultrasonic waves in Inconel super alloy. In: Proceedings of the AIP Conference, Ancona, Italy (2010)

9. Kumar, A., Shankar, V., Javakumar, T., Bhanusankara Rao, K., Raj, B.: Correlation of microstructure and mechanical properties with ultrasonic velocity in the Ni-based superalloy Inconel 625 . Philos. Mag. 82, 2529-2545 (2002)

10. Bugavev, A.A., Gupta, M.C., Pavne, R.: Laser processing of Inconel 600 and surface structure. Opt. Laser Eng. 44, 102-111 (2006)

11. Rai, S.K., Kumar, A., Shankar, V., Jayakumar, T., Bhanu, S.R.K., Baldev, R.: Characterization of microstructures in Inconel 625 using X-ray diffraction peak broadening and lattice parameter measurements. Scr. Mater. 51, 59-63 (2004)

12. Pramila, T., Shukla, A., Kishore, N.N., Raghuram, V.: Determination of elastic constants of aluminium using laser based ultrasonics. Indian J. Sci. Technol. 2, 25-28 (2009)

13. www.matweb.com (material property data)

Publisher's Note Springer Nature remains neutral with regard to jurisdictional claims in published maps and institutional affiliations. 